\title{
A PESQUISA EM HISTÓRIA DA EDUCAÇÃO - TESTEMUNHO DE UM AUTOR: ENTREVISTA COM ANTONIO VIÑAO FRAGO
}

DOI: http://dx.doi.org/10.1590/2236-3459/69016

\section{RESEARCH ON HISTORY OF EDUCATION - AN AUTHOR AS WITNESS: INTERVIEW WITH ANTONIO VIÑAO FRAGO}

\author{
Maria Helena Camara Bastos \\ Pontifícia Universidade Católica do Rio Grande do Sul, Brasil.
}

$\cos 8$

\section{Quero iniciar esta entrevista com suas memórias pessoais: como foi seu encontro e seu interesse com a História da Educação?}

La casi totalidad de los profesores e investigadores españoles en el campo de la Historia de la Educación proceden del ámbito de la Pedagogía o Ciencias de la educación, y solo unos pocos del de la Historia. En su mayor parte, pues, se formaron en la extinta sección de Pedagogía de las antiguas facultades de Filosofía, si sobrepasan los 45 años, o en las facultades de Ciencias de la educación o Pedagogía, si tienen una edad inferior. ¿Cómo es posible que alguien, como sucede en $\mathrm{mi}$ caso, cuyos estudios universitarios y doctorado tuvieron lugar en la facultad de Derecho haya terminado por recalar en el área de Teoría e Historia de la Educación, si además tampoco posee una titulación universitaria en el campo de la Historia? Eso requiere una explicación ${ }^{1}$.

Debo advertir, en primer lugar, que mis preferencias al acceder a la universidad con 16 años en el curso 1959-60 - cursé el bachillerato con uno o dos cursos de adelanto en comparación con el resto de mis compañeros - se inclinaban por el estudio, en la facultad de Letras, de la Geografía e Historia. Mi padre creyó más oportuno que me matriculara en Derecho. Entre otras razones adujo, y no le faltaba razón, que los estudios de Geografía e

\footnotetext{
${ }^{1}$ Aunque he procurado, en esta explicación, atenerme a los hechos, no está de más advertir que en estas páginas, como en todo texto autobiográfico, lo que hay es una reconstrucción y recreación del pasado desde un presente dado. Es decir, que la selección de los hechos, su conexión y su interpretación son el producto de mi actual representación mental de lo que en estas páginas se dice. 
Historia solo conducían al mundo de la enseñanza y que los profesores, en este país, no solo no eran valorados, sino que eran unos muertos de hambre. Que, por el contrario, el Derecho - no había llegado aún el tiempo de los economistas - poseía más numerosas y prestigiadas salidas profesionales. Cursé, pues, estudios de Derecho, pero si alguien observa mi expediente académico universitario, podrá ver de inmediato que las mejores calificaciones las obtuve en aquellas disciplinas de índole histórica y filosófica o pertenecientes al ámbito del derecho público.

Mis preferencias por las ciencias sociales, y no por las estrictamente jurídicas, era obvia, así que, sea por razones personales propias de la época - nada que ver con las actuales -, sea por dichas preferencias, opté por preparar las oposiciones al Cuerpo Técnico de Administración Civil, hoy Cuerpo de Gestión de la Administración Civil del Estado, cuyo temario - Derecho constitucional y administrativo, Economía, Hacienda pública, Ciencia política, Historia, Sociología - encajaba mejor en mis intereses intelectuales. Tras dos años de preparación y lecturas en estos campos y un curso de formación en la Escuela Nacional de Administración Pública, elegí destino, en 1967, en la administración periférica del Ministerio de Educación, poco antes de que se produjera el cambio de gobierno y de ministro que sería el origen de la posterior reforma educativa de 1970.

Desde septiembre de 1967 hasta septiembre de 1982 trabajé, pues, como funcionario técnico en el Ministerio de Educación y Ciencia, como gestor responsable a nivel provincial y administrativo - no político, pero en contacto con los responsables políticos de cada momento - de todo lo relativo a la puesta en marcha y aplicación de dicha reforma en unos años en los que tendría lugar, a partir de 1975, el tránsito desde un régimen dictatorial a otro formalmente democrático. Esta experiencia me sería después muy útil tanto en la docencia como en mis investigaciones en el ámbito de la educación.

Debí ser un funcionario atípico. En los primeros años de la década de los 1970 preparé por libre - es decir, como alumno no oficial y por mi cuenta, a base de lecturas algunas materias de los estudios de Sociología. Trataba simplemente de saber algo en este ámbito, no de obtener acreditaciones académicas ${ }^{2}$. Comencé, además, a preparar mi tesis doctoral en el Departamento de Derecho Constitucional o Político - donde fui profesor ayudante durante cinco años y donde trabajábamos, sobre todo, la historia de las ideas políticas y del constitucionalismo español - sobre política y educación en la España del siglo 19, con especial referencia a la enseñanza secundaria. Leí y defendí la tesis en enero de 1980. Tardé, pues, unos diez años en escribirla - en aquella época no había, en este aspecto, límites temporales; hoy no hubiera podido realizarla. Como para comprender el siglo 19 tuve que remontarme a las reformas y propuestas ilustradas del siglo anterior, y para entender estas, a Locke, Comenio y, sobre todo, al nacimiento de la

\footnotetext{
${ }^{2}$ Durante varios años, en la década de los 1970, fui profesor, en horario nocturno, de las materias de Estructura Social de España y Organización y Dirección de Empresas en la Escuela de Trabajo Social de Murcia. En la de los 1980, asimismo durante unos años, formé parte como socio fundador de una empresa privada, Espacio y sociedad, dedicada a trabajos urbanísticos y sociológicos. Y en diversas ocasiones he colaborado en algunos de los trabajos e investigaciones llevadas a cabo desde el Departamento de Sociología e Historia Económica de la Universidad de Murcia. Valgan estas referencias para entender mi enraizamiento en las ciencias sociales.
} 
ciencia moderna y la revolución educativa de los siglos 16 y 17 , entre otros temas, puedo decir que mi primera formación histórico-educativa procede de la tesis doctoral, y que tuvo lugar al margen de los circuitos de la historia de la pedagogía académica, sin que eso signifique que desconociera lo que en ellos se producía.

Si se analiza mi producción escrita en la década de los 1970, se verá, no obstante, que la mayoría de las primeras publicaciones pertenecen al ámbito de la sociología, administración y política educativa con aportaciones y enfoques históricos. Seguía moviéndome todavía en el campo de las ciencias sociales, pero ya, dentro de ellas, en las ciencias de la educación. Mi incorporación a la recién creada sección de Pedagogía de la Facultad de Filosofía, Psicología y Pedagogía de la Universidad de Murcia, y poco después al Instituto de Ciencias de la Educación de dicha universidad, tendría lugar en 1979. Primero, a tiempo parcial, y desde 1982 con dedicación completa tras pedir la excedencia en la administración pública. En dicho año comencé, además, a asistir a los congresos de la Ische y a los recién inaugurados coloquios nacionales de Historia de la Educación ${ }^{3}$. Dado que, al menos en Murcia, se me conocía más como gestor de la educación que como historiador, en un principio se me asignaron disciplinas tales como Organización y Dirección de Centros Docentes, Educación Comparada y Política y Legislación Educativa. Materias que fui dejando paulatinamente para pasar a enseñar otras de índole histórico-educativa.

En síntesis, mi formación básica procede del ámbito de las ciencias sociales, y mi dedicación a las ciencias de la educación y, dentro de ellas, con preferencia a la Historia de la Educación, es el resultado de la confluencia de mi experiencia en el Ministerio de Educación, de la tesis doctoral y de las primeras investigaciones que llevé a cabo en el Instituto de Ciencias de la Educación de la Universidad de Murcia a partir de 1979. Como toda formación inicial, mi llegada a este campo ofrecía muchas lagunas, propias de la época, del contexto y del autodidactismo que caracteriza a quienes nacimos en España en las décadas de los 1940, 1950 y parte de los 1960, a causa de las nefastas consecuencias educativo-culturales de la guerra civil y del franquismo. Lagunas que he procurado llenar en años posteriores, no siempre con éxito y sabiendo ya que, en algunos temas y materias, nunca van a ser cubiertas.

Una consideración final: no es usual que alguien finalice trabajando en un campo académico y de investigación que no se corresponde con su titulación académica formal. Además, cuando eso sucede, lo habitual es que se produzcan actitudes de recelo o rechazo por parte de quienes ya trabajan y se han formado en dicho campo. Tengo que decir que en mi caso no las hubo, o que fueron tan aisladas que no llegué a tener noticia de ellas. Al contrario, desde el primer momento siempre hallé una grata acogida y disposición en la práctica totalidad de los que, con el tiempo, serían no ya colegas y compañeros de trabajo, sino amigos.

\footnotetext{
${ }^{3}$ Sobre esta fecha emblemática para la comunidad de historiadores de la educación españoles, remito a lo dicho en Antonio Viñao, From dictatorship to democracy: history of education in Spain, Paedagogica Historica, v. 50, n. 6, 2014, p. 830-843 - la version en lengua española aparecerá en el libro de homenaje a Juan Luis Guereña actualmente en prensa. Sobre estos años, y las décadas posteriores, se encontrará información adicional en Raimundo Cuesta y Julio Mateos, Conversación con Antonio Viñao, Con-Ciencia Social, v. 2, 2002, p. 89-107.
} 
Quais temas de pesquisa inicialmente tiveram a sua atenção? Quais estudiosos influenciaram sua formação historiográfica?

Intentaré responder de modo conjunto a ambas cuestiones, así como a las preguntas formuladas en los dos epígrafes siguientes por la estrecha relación que existe entre dichas cuestiones y preguntas. Dejo a un lado los trabajos de política y legislación educativa o de sociología de la educación, aunque en ocasiones mencione autores de estos ámbitos que también han influido en mi formación. De todas formas, como síntesis anticipatoria, copio el párrafo que figura en una de esas notas biográfico-académicas que de vez en cuando nos piden: sus campos de investigación prioritarios son la historia de la alfabetización - la lectura y la escritura como prácticas sociales y culturales -, de la escolarización y de la enseñanza secundaria, así como la historia del currículum espacios y tiempos escolares, disciplinas, libros de texto - y la de la relación entre las culturas escolares y las reformas educativas. A esta sintética relación habría que añadir, en los últimos años, otra línea de investigación: la relativa a la memoria escolar y el patrimonio educativo.

En mi tesis doctoral me fueron útiles, por ejemplo, la obra de Durkheim sobre la evolución pedagógica en Francia, y la de un sociólogo francés no muy conocido, Edmund Goblot, sobre la educación secundaria en el país vecino y sus relaciones con los cambios sociales: pura sociología histórica. Y, en relación con España, algunos de los trabajos de Julio Ruiz Berrio sobre los siglos 18 y 19, otros estudios histórico-educativos de autores procedentes del campo de la historia del derecho - Antonio Álvarez de Morales, Mariano Peset -, o de la ciencia - José Luis Peset, José María López Piñero -, y sobre todo una obra, La educación y la escuela en España de 1874 a 1902, escrita por una historiadora francesa, Ivonne Turin, que me descubrió la conflictiva historia educativa de mi país. La fecha de adquisición del ejemplar, profusamente subrayado, que obra en mi biblioteca, 20 de enero de 1969, era toda una premonición de lo que vendría después.

De un modo u otro, fueron años de muchas lecturas en muy diversos ámbitos y no resulta fácil detectar influencias que muchas veces revisten un carácter difuso. Menciono solo un ejemplo: en aquellos años me interesé, entre otros temas, por la historia de la ciencia y la técnica - Bernal, Rossi, Butterfield, Mumford, Garin -, así como por sus relaciones con una concepción científico-técnica de la educación en general y de la enseñanza media en particular. Pese al buen número de libros del que he tenido que desprenderme, por unas u otras razones, a lo largo de mi vida, veo que en mi biblioteca particular hay todavía cinco libros de Benjamin Farrington, un autor hoy olvidado o poco leído que, sin embargo, me hizo ver el mundo clásico y, por tanto, el humanismo renacentista y los orígenes de la ciencia moderna desde otra perspectiva. ¿Hasta qué punto influyó en mi tesis doctoral y en otros escritos de los años 1970 y 1980 ? Lo ignoro. Lo que sí sé es que la lectura de sus obras, y de otros autores, me hace hoy todavía desconfiar de quienes se apropian del término humanismo para reducirlo a una visión exclusivamente centrada en lo que se ha dado en llamar el mundo de las letras; es decir, de quienes separan el cerebro de las manos.

Tras la tesis, todo indica que a comienzos de los 1980 mis intereses comenzaron a desplazarse hacia la historia social y cultural de la educación. La asistencia a los congresos de la Ische y los coloquios de Historia de la Educación de Tours/Francia, los 
trabajos del Seminari d'Historia de l'Ensenyament creado en Barcelona (1973-1980) al estilo de los history workshop ingleses, la lectura de algunos autores como De Vroede, Frijhof, Julia, Chartier, Compère, Guereña o Brian Simon, con algunos de los cuales colaboraría más tarde, así como los cambios que, a partir de 1982, comenzaron a operarse en la historiografía educativa española, me confirmaron que iba en la dirección correcta. Precisamente esto fue lo que me condujo, junto con el conocimiento de parte de buena parte de la producción sobre la historia de la alfabetización y de la cultura escrita que se estaba publicando fuera de España, a mis investigaciones sobre este tema en la primera mitad de la década de los 1980.

No está de más advertir que desde el principio me negué a distinguir entre historia social e historial cultural, como me he negado a considerar compartimentos estancos lo cuantitativo y lo cualitativo, lo global, lo nacional y lo local, o lo macro, lo medio y lo micro. Se trata distinciones académicas y formales. La realidad siempre es una combinación o mezcla de aspectos y elementos de un tipo y otro. De ahí que sea imposible atender a unos sin tener en cuenta los otros.

La historia de la alfabetización la abordé, pues, cuantitativa y cualitativamente. Por un lado, influido por las investigaciones de Harvey J. Graff y los autores franceses, Furet y Ozouf, e ingleses, Stone, así como por hispanistas - Bennassar, Nalle, Saugnieux, Soubeyroux - que trabajaban estos temas con una perspectiva desde luego cuantitativa pero también, sobre todo, socio-cultural e ideológica. Por otro, leyendo a quienes desde la psicología transcultural y la antropología se interesaban por los procesos de alfabetización y sus consecuencias cognitivas y socio-culturales: Goody, Olson, Scribner, Luria. La lectura en 1982 de Orality and literacy de Walter J. Ong, el mismo año de su publicación, me abrió la mente - pese a disentir de algunas de sus interpretaciones- , a perspectivas y enfoques por mí poco conocidos, así como al resto de su obra. Asimismo, me puso en contacto con la Escuela de Toronto - Havelock, McLuhan, Innis - y me llevó a interesarme por la historia de la lectura y la escritura como prácticas socio-culturales y la historia de la cultura escrita y de las recepciones y apropiaciones de lo escrito, un campo en el que debo destacar mis deudas con Roger Chartier, Anne-Marie Chartier, Armando Petrucci, Jean-François Botrel, Neil Postman, Víctor Infantes, François Lopez y Antonio Castillo, entre otros autores.

Sea porque el tema de la alfabetización me llevara al de sus relaciones con la escolarización, sea porque mi experiencia en el ministerio de Educación me había puesto en contacto con las cuestiones relativas a la arquitectura y el espacio escolar; sea, en fin, porque durante algunos años me encargué de la docencia de la asignatura de Organización y Dirección de Centros Docentes, el hecho es que en la segunda mitad de la década de los 1980 comencé a trabajar, por un lado, la historia de la escolarización, asimismo en su doble dimensión cuantitativo-estadística y socio-cultural, y la de la organización del espacio y el tiempo escolares, el paso de la escuela-aula a la escuela- 
colegio o graduada y las relaciones internas entre el profesorado y la dirección ${ }^{4}$. El resultado de estas investigaciones fueron una serie de publicaciones que aparecerían, en España y en otros países, en la década de los 1990.

Algunas de dichas investigaciones formaron parte de la memoria que presenté, en 1996, para acceder a la cátedra de Teoría e Historia de la Educación. Una memoria que organicé en torno a cuatro capítulos básicos: el primero, sobre la importancia y utilidad de la Historia de la Educación, y los tres restantes sobre cada uno de los elementos básicos de toda actividad humana: el espacio, el tiempo y el lenguaje o modos de comunicación. Desde una perspectiva general, no histórico-educativa en un sentido estricto, dichas publicaciones muestran la influencia, entre otros autores, de buena parte de la escuela francesa de Annales - Le Goff, de quien he sido un asiduo lector, y Duby sobre todo -, de Elias, Burke, Certeau y Ricoeur, así como de antropólogos como Goody, Hall o Geertz. O, ya en el ámbito histórico-educativo estricto, de autores como Hamilton, Depeape, Frank Simon y Escolano, que en esos mismos años se preocupaban por entrar, desde la historia, en la caja negra de la escuela. En cuanto al tema del lenguaje o discurso histórico - era el tiempo de las polémicas con motivo de la obra, entre otras, de Hayden White - aprendí mucho leyendo, asimismo entre otros, a Chartier, Barthes, Darnton, Fish, Ginzburg, a los autores de la estética de la recepción - Jauss, Iser - y, aunque parezca un poco extraño, a los escritores del grupo Oulipo, en especial a Perec y Calvino.

Los primeros trabajos en cuyo título utilizo la polisémica expresión de cultura escolar son de la segunda mitad de la década de los 1990. Nada extraño, se dirá, en alguien que se movía en el ámbito de la historia cultural. En mi caso, sin embargo, llegué a ella desde al menos cuatro direcciones.

Por un lado - combinando, en este caso, experiencia personal y lecturas - , desde el análisis de las resistencias, continuidades e inercias del sistema escolar a las reformas e innovaciones educativas, un aspecto en el que me fue muy útil la lectura de autores como Cuban, Tyack y Sarason.

Por otro, desde el intento, ya indicado, de entrar en la caja negra de la escuela y, por tanto, en la vida cotidiana de los centros docentes, en el día a día de las aulas a partir de nuevas fuentes y nuevas miradas sobre fuentes ya conocidas, con especial atención, siempre, por las relaciones entre los saberes y lo poderes. Es decir, por el análisis micropolítico de las instituciones educativas.

Por otro, desde mis primeros trabajos sobre el proceso de profesionalización docente en España, un tema en el que debo también mucho a los trabajos de Antonio Nóvoa. La formación de los cuerpos docentes en la España de finales del siglo 18 y comienzos del 19 me llevó al tema de las disciplinas y saberes académico-escolares, y

\footnotetext{
${ }^{4}$ En la lectura de la tesis doctoral en 1980, uno de los miembros del tribunal, Francisco Rubio Llorente, más tarde presidente del Tribunal Constitucional, hizo una acertada crítica de la misma: ¿cómo era posible estudiar la génesis y evolución de la segunda enseñanza en España sin dedicar un capítulo específico al profesorado? Había, en efecto, alusiones y observaciones aisladas al tema, pero faltaba un tratamiento de conjunto de una cuestión clave en dicha génesis y evolución. Rubio Llorente achacó dicha carencia al sesgo propio de quien, en aquella época, era un funcionario técnico de la administración educativa: mi perspectiva era más político-administrativa que pedagógico-educativa. Una vez ubicado profesionalmente como profesor universitario mi perspectiva cambió: el tema del profesorado, el de su formación y profesionalización, se ha convertido, sobre todo en las dos últimas décadas, en uno de los campos de investigación a los que he dedicado una atención preferente. He ahí un ejemplo más de cómo el lugar desde el que uno mira condiciona lo que ve y como lo ve.
} 
desde este último, a través de las obras de Chervel y Goodson y, en España, del grupo Nebraska: Raimundo Cuesta, Juan Mainer, Julio Mateos, con cuyos componentes siempre he mantenido una fructífera y amistosa conversación e intercambio intelectual, a interesarme por las continuidades y discontinuidades entre los cambios políticos y los cultural-educativos $y$, en relación con ello, por las periodizaciones históricas que manejamos.

Por último, para entender los sistemas educativos - en cuyo análisis me considero deudor, entre otros autores, de los trabajos de Archer, Green, Puelles, Müller, Ringer, Simon, Tiana y Ossenbach - , su estructura y procesos, y las tendencias y fuerzas internas que genera su misma existencia. Estructura, procesos, tendencia y fuerzas que constituyen el contexto o nicho en el que nacen, se configuran y operan las diferentes culturas escolares.

Nunca, debo aclararlo porque no siempre se me ha entendido así, he mantenido que dicho concepto, el de cultura escolar, y otros con él relacionados - código disciplinar, gramática de la escolaridad, etc. -, sean la clave para explicar todos los cambios y continuidades en el currículum y en la organización escolar. Sirven - nos han servidos a mí y a otros - para explicar, como he dicho, el fracaso relativo de las reformas e innovaciones educativas, una parte - solo una parte - de lo que sucede en la vida cotidiana de los centros docentes y aulas, la génesis y evolución de determinadas disciplinas, saberes y actividades escolares, o sus relaciones con los sistemas educativos en los que anidan. Pero no son conceptos o enfoques explícalo-todo. Entre otras razones, porque no hay conceptos o enfoques que lo expliquen todo. En los últimos años, visto el uso y abuso que se viene haciendo del concepto de cultura escolar - todo es cultura - , soy más cauteloso y reacio a utilizarlo, y cuando lo utilizo lo hago en plural, culturas escolares.

En las dos últimas décadas pueden observarse, en las líneas de investigación seguidas, la influencia de los giros visual y memorialístico-patrimonial. No es posible sustraerse al signo de los tiempos, aunque sean revisitados temas de épocas anteriores la historia de la lectura y la escritura, en especial sus aprendizajes; el proceso de profesionalización docente; el espacio y el tiempo escolares; la política educativa y el papel de los poderes públicos en la educación; etc. - y se aborden otros nuevos, como el republicanismo cívico-educativo, no de moda en la historiografía educativa pero sí en la del pensamiento e ideas políticas. En último término, no es posible referirse a lo visual o lo patrimonial sin tener en la mente o acabar refiriéndose a la memoria. Hay en el fondo en mi producción más reciente, de forma expresa o no, más de lo memorialístico que de lo patrimonial o icónico-visual. Debe ser cuestión de la edad o, también de mi interés, como investigador y lector, por los sujetos - el retorno del sujeto - , los procesos de subjetivación y el género autobiográfico y diarístico en todas sus manifestaciones, de modo especial entre el profesorado y el alumnado. Baste decir que mi biblioteca particular cuenta con unas 300 obras de este tipo. Las autobiografías, memorias y diarios son una de mis lecturas preferidas, con independencia de la relevancia del autor o, incluso, de su calidad literaria.

Es posible que en lo dicho hasta ahora se adviertan algunas ausencias o se echen de menos algunas referencias o influencias intelectuales. Por ejemplo, a los enfoques marxistas. En los 1960 y 1970 fui un asiduo lector de lo que entonces se llamaba el 
humanismo socialista ${ }^{5}$, más centrado en los escritos del joven Marx, sobre todo en sus Manuscritos económico-filosóficos ${ }^{6}$, que en la jerga escolástico-marxista explícalo-todo de Althusser, Poulantzas o Harnecker. Admiré, y admiro, al Marx analista de la realidad y de la política de su tiempo. Sus artículos periodísticos y escritos sobre la España del siglo 19 recuerdo que me sorprendieron por su lucidez y capacidad para saber ver lo que otros no vieron pese a tenerlo ante sus ojos.

Pero no soy un marxista a ultranza, como tampoco un foucaultiano a ultranza, aunque agradezca a Foucault que nos haya desvelado, entre otras cosas, los mecanismos que explican lo que Étienne de la Boétie llamó la servidumbre voluntaria. Por supuesto, ello no quiere decir que no me sirva de ambos, y de otros pensadores, cuando veo, como muchas veces sucede, que sus conceptos, ideas y enfoques arrojan alguna luz sobre lo que deseo analizar. En el fondo, si soy algo a ultranza, sin reticencias ni dudas, es en la defensa de la libertad intelectual y de pensamiento y, correlativamente, del nomadismo entendido como no encasillamiento unidireccional en una u otra corriente, autor o tendencia. Es decir, del perspectivismo metodológico; de la idea de que todo objeto o asunto contemplado desde diferentes posiciones deja de ser el mismo objeto o asunto. Eso no quiere decir que todas las miradas sean válidas - las hay ciegas o que ocultan más de lo que muestran -, sino que no hay ninguna que nos ofrezca una explicación o visión total. $\mathrm{O}$, si se prefiere, que un intento de explicación total requiere diversas perspectivas.

Más influencia tuvieron en mis investigaciones, al menos eso es lo que me parece ahora, determinadas lecturas de sociología de la educación efectuadas en las décadas de los 1970 y 1980. En especial de Bourdieu - recuerdo la impresión que me produjo Les héritiers -, Bernstein, Lerena y Varela. La vida intelectual tiene épocas, modas y descubrimientos. Esa fue una época, lo mismo que en esta me atrae, por poner algunos ejemplos, leer a Nietzsche, Steiner, Bunge, Wagensberg, o a todo aquello que me aclare cómo a partir de la vida y entorno de un judío ágrafo que se atribuía, o le atribuyeron, cualidades proféticas - es decir, del judaísmo- surgieron, y surgen todavía, cientos de cristianismos, unos vencedores y pujantes y otros en germen, derrotados o extintos. Estos son intereses, por así decir, intelectuales, aunque tengan también algo de personales. Algo distinto de la impresión o huella que me han dejado la vida y obra de unos pocos personajes.

\footnotetext{
${ }^{5}$ Este sería el título de una obra colectiva, editada por Paidós en 1966, que sigue en mi biblioteca - fue adquirida el 14 de febrero de 1967 y también se halla profusamente subrayada - en la que colaboraban Suchodolski, Goldmann, Senghor, Marcuse, Schaff, Bloch, Fromm, Russell, Bottomore, Medow, y della Volpe, entre un buen número de intelectuales, economistas y sociólogos procedentes sobre todo de los países del Este de Europa. Eran los años en los que, frente modelo soviético, había quienes se inclinaban por un supuesto modelo yugoeslavo de vía hacia el socialismo, basado en la autogestión colectiva, el cooperativismo y la planificación descentralizada, que ignoro si alguna vez llegó a tener vigencia. Dicho modelo combinaba además bastante bien, al menos teóricamente, con el de quienes entendían que era posible la coexistencia, en los países europeos de economía capitalista y socialdemócrata, de un sector privado, sujeto a las condiciones del mercado, con un sector de empresas públicas competitivo y económicamente rentable que sirviera de contrapeso. Todo eso ha saltado por los aires. Por cierto, el trabajo que presenté en el período de formación llevado a cabo en la Escuela Nacional de Administración Pública, en el curso 1966-67, versó sobre El control de las empresas públicas en Francia.
}

${ }^{6}$ Fecha de adquisición: 7 de junio de 1968. Hist. Educ. (Online) $\quad$ Porto Alegre 
En el historiador hay que distinguir dos aspectos que a veces se confunden. Una cosa es la influencia intelectual de determinadas obras o autores y otra la impresión personal que deja o produce el conocimiento de su vida y sus obras. Si en mis años jóvenes, los 1960, me impresionó, por ejemplo, Simone Weil por su coherencia, o por su búsqueda de la coherencia entre pensamiento y acción, recientemente he vuelto a interesarme por ella ${ }^{7}$, y en los 1970 ese joven Gerald Brenan capaz de dejar el selecto y londinense Círculo de Bloomsbury y perderse en una aldea de la Alpujarra granadina para escribir El laberinto español o sobre la poesía de san Juan de la Cruz, en los años 1980 y 1990 me atrajo con tal intensidad la figura de Blanco White, por su tenaz, insobornable e infructuosa búsqueda de su verdad, y leí y escribí tanto sobre su vida y su obra, que a veces tengo la impresión de que forman parte de la mía.

Hechas estas aclaraciones, que creí necesarias, paso a referirme al contexto historiográfico - espacio-temporal - en el que se ha desarrollado mi obra y que, por tanto, explica buena parte de la misma. Pertenezco a esa generación de historiadores de la educación que en la España de los años 1980 del siglo pasado constituyó un campo académico y científico que buscaba, al mismo tiempo, un espacio propio e independiente en el campo pedagógico y un reconocimiento científico en el de la historia en general y en el de la Historia de la Educación a nivel internacional. No fue fácil y tampoco se consiguió por entero. Ese no es el tema ahora. Lo que intento decir es que buena parte de mi producción científica y actuaciones solo se entienden en dicho contexto y teniendo en cuenta el intercambio de ideas, enfoques, lecturas, etc. con historiadores de la educación con los que he compartido muchas horas y conversaciones.

En España, y entre los de esa primera generación, con Julio Ruiz Berrio, Agustín Escolano, Manuel de Puelles, Aida Terrón, Alejandro Tiana, Gabriela Ossenbach, José María Hernández, Antón Costa, Herminio Barreiro, Narciso de Gabriel, Buenaventura Delgado, Juan Luis Guereña, Eugenio Otero, Alejandro Mayordomo, Pere Solà, Jordi Monés, Bernat Sureda, Carmen Sanchidrián, Luis Miguel Lázaro, Juan Manuel Fernández Soria, Pedro Álvarez y un largo etcétera en el que incluyo a muchos colegas que espero no se molesten por no haber sido expresamente mencionados. No aludo a los de la generación posterior, la relación sería también muy extensa, salvo a María del Mar del Pozo por los viajes compartidos a la Ische y por lo mucho que he aprendido de ella, y espero seguir aprendiendo. La pertenencia a la comunidad académico-científica de historiadores de la educación españoles explica, en primer lugar, la atención prestada a la Historia de la Educación de mi país - intentando situarla siempre, de forma expresa o no, en un contexto europeo e internacional - , y, en segundo lugar, a determinados temas, épocas y autores - la Institución Libre de Enseñanza, la II República, etc. - que fueron objeto de atención preferente por dicha comunidad en las años finales del siglo pasado y los comienzos de este. No obstante, me sigue apasionando una época que los jóvenes historiadores de la educación española consideran ya muy alejada en el tiempo y, por tanto, de sus intereses. Me refiero a las últimas décadas del siglo 18 y el tránsito del Antiguo Régimen a un liberalismo nunca bien asentado. Ahí es donde reside el origen de nuestras desgracias.

\footnotetext{
7 Todavía conservo el primer libro que adquirí de Simone Weil: La pesanteur et la grâce. Lugar y fecha de adquisición: Lyon, 29 de agosto de 1962. 
A nivel internacional, además de las influencias ya mencionadas - Depaepe, Simon, Cuban - he leído también con provecho y profusión a autores como Richard Aldrich, Gary McCulloch, Ian Grosvenor, Jeroen Dekker, David Labaree, Pierre Caspard, Antoni Santoni Rugiu, $M^{a}$ Esther Aguirre o Rubén Cucuzza, por citar solo algunos de aquellos que considero más próximos generacional e intelectualmente.

Por último, una parte de mi producción historiográfica solo se explica conociendo el contexto académico más próximo: la Facultad de Educación de la Universidad de Murcia; dentro de ella el Departamento de Teoría e Historia de la Educación y, más al interior todavía, el grupo de investigación Educación, sociedad, historia y el Centro para el Estudio de la Memoria Educativa - Ceme. Mis aportaciones en el campo de la historia de las disciplinas escolares no hubieran sido posibles, o hubieran sido diferentes, sin la serie de tesis doctorales sobre la historia de la enseñanza de las matemáticas, la física y química, las ciencias naturales, el dibujo, la música, la historia o la declamación teatral, entre otras materias o saberes, dirigidas en dicha facultad y llevadas a cabo por profesores de los mencionados ámbitos disciplinares, o que pertenecen a distintos campos de las didácticas específicas. De igual modo, los proyectos llevados a cabo por el grupo de investigación en las dos últimas décadas en el ámbito memorialístico, patrimonial y museístico de la educación, y los trabajos elaborados en el mismo, solo se entienden como una labor de grupo y como resultado de conversaciones y tareas compartidas en las que Pedro Luis Moreno, actual responsable del grupo de investigación tras mi jubilación, ha desempeñado un papel relevante.

\section{Em um balanço de sua trajetória de pesquisa, que contribuições destacarias?}

La pregunta queda contestada en el punto anterior, pero sintetizo la respuesta. Las contribuciones a destacar se han producido en el ámbito de: la Historia de la Educación secundaria, la de la cultura escrita y los procesos de alfabetización y escolarización, la de la escuela como institución - organización escolar, espacios y tiempos -, la de la cultura y disciplinas escolares en sus relaciones con las reformas educativas, y la de la memoria y el patrimonio educativo. No obstante, repito lo dicho: estas contribuciones solo se entienden en conversación con colegas españoles y de otros países, y son el producto de muchas lecturas en muy diversos campos, que no siempre se reflejan de modo directo en los textos escritos. Soy un lector empedernido que, además, bebe en muy diversas aguas. Un claro hijo de la cultura de la imprenta y del libro impreso, medio adaptado a la cultura electrónica.

En fin, si hay algo que caracteriza dichas contribuciones es que todas ellas han tenido lugar o se han movido en zonas fronterizas a la Historia de la Educación. Visto desde fuera, y con cierta magnanimidad, pienso que mi principal contribución ha sido la de, junto con otros, abrir la Historia de la Educación a otros campos historiográficos historia socio-cultural, historia de la cultura escrita, historia de la ciencia, historia literaria, historia sin más adjetivos - y científicos - sociología, antropología, psicología, ciencia política, derecho, filosofía - y bucear en los límites y en los espacios fronterizos, unas veces con relativo fruto y otras sin él. 
Durante as últimas décadas da História da Educação como área de pesquisa tem sido submetida a uma significativa mudança de paradigma. Como essa mudança se refletiu em seus caminhos e temas de investigação?

En general, los cambios de paradigma en la Historia de la Educación han sido una consecuencia tardía, con cierto retraso, de los acaecidos en las ciencias sociales y, dentro de ellas, en la historia. El paso desde la tradicional historia de las ideas o del pensamiento, de signo esencialista, atemporal y hagiográfico, y de una asimismo descontextualizada historia de las instituciones educativas, a la historia social, primero, y después, sucesivamente, a la historia cultural y a la historia postmoderna con los giros lingüístico, visual y memorialístico, puede desde luego apreciarse en la evolución de los temas y enfoques de mi producción escrita. Pienso, no obstante, que, aunque eso sea cierto, también lo es que no se trata de cambios bruscos, sino más bien de transiciones suaves, a veces naturales, así como de superposiciones e hibridaciones entre unas y otras tendencias. Se abren progresivamente nuevos campos, pero no se cierran los anteriores. Permanecen ahí, reaparecen, son revisitados, pero no desaparecen.

Recentemente, assumiste a função de editor da revista Historia y Memoria de la educacion da Sociedade Espanhola de História da educação. Poderias comentar como tem sido essa experiência?

En efecto, fui nombrado director de Historia y Memoria de la Educación por la Sociedad Española de Historia de la Educación - Sedhe - con el encargo, junto con el Consejo de Redacción, de poner en marcha la revista científica de dicha Sociedad. Una revista bianual, electrónica y de libre acceso. El primer número apareció en marzo de 2015, a finales de 2016 aparecerá el número cinco, y en estos momentos tenemos programados el tema monográfico de todos los números de 2017 y 2018 y el primero de 2019.

Mi corta experiencia al frente de la revista es algo contradictoria desde un punto de vista personal. Es obvio que no es la revista de Antonio Viñao - como en algún caso he tenido que escuchar -, sino de la Sedhe, entre otras razones porque mi nombramiento como director tiene un límite temporal. Es una revista institucional que, por tanto, debe intentar reflejar la pluralidad de enfoques, temas, ideología, tendencias, etc., que existen en la Sedhe. Además, es conocido que la revista nació tras una serie de conflictos internos que originó que dicha sociedad se desvinculara de la revista Historia de la Educación, editada por la Universidad de Salamanca. Y, por si ello no fuera suficiente, el contexto académico-científico en que nació, se caracteriza por una creciente internacionalización de las revistas científicas - que algunos confunden con anglosajonización - , la difusión y el acceso electrónico, una presión cada vez mayor por publicar en revistas de impacto de acuerdo con unos criterios a mi juicio puramente formales y burocráticos - un reconocimiento que las nuevas revistas no pueden alcanzar hasta al menos cinco o seis años de existencia y, en más de un caso, previo pago de una cantidad determinada -, y la progresiva mercantilización del mundo de las revistas académico-científicas; es decir, la irrupción del capital en un sector en el que hay beneficios económicos y un poder académico-científico-cultural a disputar. Una 
mercantilización que nos convierte extrañamente, por propia voluntad, en mano de obra gratuita de corporaciones empresariales que obtienen, gracias a nuestro trabajo, un determinado beneficio económico. Así, evaluamos, informamos y escribimos para dichas corporaciones sin percibir retribución alguna, y se nos recompensa figurando en consejos de redacción, certificando nuestra tarea como evaluadores, publicando el producto de nuestro trabajo, con prestigio académico, etc.

Dirijo, por tanto, una revista institucional que busca integrarse en ese nuevo mundo de las revistas científicas, no el tipo de revista que me gustaría crear, junto con otros investigadores sociales, al margen de las exigencias formales, las presiones externas y la creciente mercantilización indicadas.

La nueva situación tiene, desde luego, aspectos positivos. La internacionalización obliga a aceptar artículos en al menos aquellas lenguas que tienen una mayor difusión. En el caso de Historia y Memoria de la Educación se aceptan, por ejemplo, textos en español, portugués, italiano, inglés y francés. Asimismo, la edición electrónica permite, por su bajo coste, que se incluyan versiones de un mismo trabajo en diferentes idiomas siempre y cuando al menos uno de ellos sea de los admitidos oficialmente. Eso quiere decir, por poner tres ejemplos, que se admiten artículos con versiones en español y catalán, en portugués e inglés o en inglés y húngaro. Esta posibilidad plantea un problema, toda nueva situación, abre nuevos problemas: el de asegurar que el texto escrito en un idioma que no es el de quien lo escribe, es lingüísticamente aceptable: incluso, en bastantes ocasiones, aunque se escriba en el idioma propio. Un hecho que se complica cuando se opta por publicar en inglés y no en la propia lengua. Por cierto, ¿cuál es la razón por la que se valora más un artículo escrito en inglés que en cualquier otro idioma? He ahí una cuestión que dejo en el aire.

Está fuera de toda duda que la edición electrónica ha permitido la existencia de un mayor número de revistas - lo que, al menos teóricamente, aumenta la competencia entre ellas y la calidad de lo publicado -, y que algunos de los requisitos formales que se exigen tienen por finalidad asegurar dicha calidad y la objetividad de las decisiones que se toman. Así sucede, por ejemplo, con los dos informes ciegos. Otras exigencias formales por ejemplo, que figure tal o cual dato en este u otro lugar - no pasan de ser requisitos burocráticos sin relación alguna con la mencionada calidad.

Sin embargo, estos aspectos positivos tienen también efectos no deseables y previsibles, aunque no previstos, y desde luego en este momento imparables. En cuanto a la proliferación de revistas - en un campo como el de la Historia de la Educación en el que no solo hay que estar atento y conocer lo que se publica en los tan fragmentados mundos de la historia y de la educación o pedagogía, sino también en otras ciencias sociales y humanas fronterizas, y no menos fragmentadas -, se corre el peligro de que dicha proliferación, consecuencia asimismo del publicar o morir, haga que sea tal el número de revistas digitales o impresas - accesibles también, por lo general, por vía electrónica- que se reciben, que, cuando llegan por una u otra vía, nos limitamos a leer el índice con los autores y títulos de los artículos, ojear, como mucho, las reseñas de libros, y guardarlas o archivarlas en el lugar físico o virtual correspondiente. Eso si esta última operación, la de su guarda y archivo, no se produce de modo inmediato tras la recepción de la revista. Además, añado, están comenzando a proliferar espacios electrónicos y redes sociales de colaboración e intercambio científico que, en el fondo, no son sino versiones ultrarrápidas, 
casi instantáneas, de las revistas científicas. ¿Para qué esperar varios meses a publicar un texto recién escrito, o intervenir en un debate a través de una revista, cuando puedo difundirlo de inmediato? ¿Cómo se combinarán en el futuro ambos ámbitos, el de las revistas y el de las redes o espacios electrónicos, y cómo se articularán entre sí? ¿Qué modificaciones supondrá ello en el mundo de la escritura y lectura científicas? ¿Dejaremos algo para los libros? ¿Habrá tiempo para escribirlos?

Más revistas no significan pues, necesariamente, más competitividad y más calidad. Lo que sí significa son más textos no leídos, o ni siquiera conocidos, más publicar por publicar, y más posibilidades, dada la rápida obsolescencia de lo que se escribe, de que la calidad global de los trabajos sea la que corresponde a un sistema que no deja lugar a la reflexión y la lectura o escritura sosegadas.

La cuestión de los dos o más informes ciegos a que deben someterse los artículos para ser aceptados, es una de las más delicadas en la dirección de cualquier revista. Su finalidad es clara: asegurar la calidad y objetividad en las decisiones que se adopten. La práctica, según mi breve experiencia, es más compleja. La elección de los informantes puede determinar en cada caso, con un margen de error mínimo, los resultados del informe. Elegirlos no es una cuestión simple y los criterios a tener en cuenta en dicha elección son diversos: tema, citas, relaciones personales, filias y fobias académicas, idioma, autor novel o de reconocido prestigio, etc. Además, se aprecia en los informes una cierta tendencia a la admisión del artículo evaluado con unas pocas modificaciones, en su mayor parte formales, o al rechazo sin más. El término medio - el informe favorable pero que exige, con el fin de mejorar el trabajo, modificaciones relevantes y lecturas que se detallan - requiere más tiempo y dedicación por parte del informante, y no siempre es bien recibido, como debiera serlo, por el autor o autora. Por otra parte, al menos en Historia y Memoria de la Educación, es posible, y positivo, que quien envía un artículo reciba, además, de las recomendaciones de los informes ciegos, una serie de propuestas de modificación y mejora procedentes del Consejo de Redacción. Queda claro, en todo caso, que esta es una cuestión, la de los informes ciegos que requiere flexibilidad, un conocimiento del campo de investigación, de quién es quién en el mismo, y un buen trato, por no decir, diplomacia en la comunicación con autores e informantes.

La flexibilidad, asimismo, es un criterio a seguir en relación con la estructura o partes de la revista, la composición de cada número, la aplicación de las instrucciones para los autores, y la misma disposición o despliegue interno de los textos. Eso no quiere decir que no existan criterios o normas, o que existan y se apliquen de modo arbitrario o discrecional. Quiere decir que siempre habrá situaciones imprevistas, que lo que importa no son las formas, sino el contenido, y, sobre todo, que dichos criterios o normas no pueden llegar, bajo el influjo de las ciencias llamadas duras - como en más de un caso sucede en el campo de la educación -, a exigir a los artículos: a) un número de palabras reducido; b) un sistema, el APA, que permite incluir referencias bibliográficas sin fin y sin indicación de la página o páginas relativas al tema de que se trate; y c) una estructura formal, semejante para todos los artículos, que más o menos siga el siguiente orden: objetivos, metodología, datos e información básica, resultados y conclusiones.

Una breve y clara referencia, por último, a los índices de impacto de las revistas medidos en función del número de citas que reciben los textos que publican. No entro ya en los intereses económicos, y de poder, que hay detrás de dichos índices y bases de 
datos. Me limito a indicar que no miden la calidad, sino solo aquello que miden. $O$ sea, el número de citas, con todas las ponderaciones posteriores que quieran hacerse, y ello en relación con unas revistas determinadas de un ámbito territorialmente restringido, de un campo limitado - en el de la Historia de la Educación solo se tienen en cuenta, al menos en España, las revistas pedagógicas y no las de historia - y con exclusión de soportes tan relevantes como el libro o las actas de congresos.

No contexto atual da Espanha, como você avaliaria o estado da pesquisa e da disciplina História da Educação? Segundo a sua experiência, quais as perspectivas da história da educação na Espanha?

La Historia de la Educación en España, como disciplina y campo de investigación, se halla, desde hace varios años, en fase de renovación generacional. Una gran parte de quienes, en los 1980 y 1990, contribuimos a configurar dicho campo a nivel nacional e internacional nos hemos jubilado, se jubilarán en los próximos años o, desgraciadamente, han fallecido. Tras dicha generación viene otra, que también participó en esa configuración del campo, con capacidad suficiente para servir de puente con quienes trabajan en el mismo desde hace pocos años o están en los inicios de sus carreras académicas.

La situación es muy diferente a la que existía a comienzos de los 1980 cuando hubo que crear el campo independizándose de la pedagogía y hacer acto de presencia en los escenarios internacionales. Además, hoy el contexto académico-científico internacional y tecnológico plantea otros retos. Pero, hay problemas o cuestiones que no han variado sustancialmente. Por ejemplo, la tensión entre la naturaleza histórica del campo y su existencia académica en el más amplio campo de las ciencias de la educación o pedagogía. Y, por tanto, el que la Historia de la Educación, como campo de investigación, dependa en gran medida - por lo que se refiere a su peso, a la formación de quienes al mismo acceden y a los temas tratados-, del peso, formación y temas que tenga como disciplina académica.

Sua relação com pesquisadores no Brasil e a participação significativa em publicações, especialmente na revista História da Educação/ASPHE, sempre foi muito intensa. Como essa aproximação se deu? Como observas a caminhada brasileira no campo da História da educação?

Como ya comenté en la introducción del primer libro que publiqué en Brasil, el inicio de mi relación con la comunidad científico-educativa brasileña se produjo en diciembre de 1990 cuando recibí una carta de Tomaz Tadeu da Silva pidiéndome la autorización para traducir un artículo mío que había aparecido un año antes en la Revista de Educación, con la intención de publicarlo en Teoria \& Educação. Después vinieron los libros Alfabetização na sociedade e na historia; Vozes, palabras e textos, 1993; Curriculo, espaço e subjetividade: a arquitectura como programa, en colaboración con Agustín Escolano, 1998, diversos artículos y capítulos de libros colectivos, los viajes - uno en 1994 con diversos seminarios, conferencias y conversaciones en la Universidad Pontificia de Rio de Janeiro, las universidades estatal y pontificia de Porto Alegre y el II Congreso 
Ibero-americano de Historia de la Educación Latino-americana, celebrado en Campinas; otro en 2001 con actividades similares en la universidades estatales de Belo Horizonte y Campinas y la Pontificia de São Paulo y la conferencia de apertura del I Congreso de Historia de la Educación brasileña en Rio de Janeiro-, las estancias, más o menos prolongadas, de historiadores de la educación brasileña en Murcia, y textos como el de Marcus Aurelio Taborda de Oliveira y Luciano Mendes de Faria Filho sobre algunos aspectos relevantes de mi producción escrita ${ }^{8}$. A ello habría que añadir mis contactos en los congresos de la Ische o, en los celebrados en España, con quienes, desde Brasil, asistían a los mismos.

Toda esta serie de actividades, trabajos, viajes, etc. no hubiera sido posible sin las gestiones, el apoyo y la compañía de tantos historiadores de la educación que espero se me excuse de mencionarlos. Simplemente diré que han sido muchos y que guardo un grato recuerdo de todos ellos. También diré que esta recepción y apropiación de mis trabajos sobre la historia de la alfabetización y la cultura escrita, el espacio y el tiempo escolares, la historia cultural de la educación, la de las disciplinas, la cultura material de las instituciones educativas o la memoria escolar, entre otros temas, no ha sido unidireccional. Me explico. Lejos de mí la pretensión de estar al día en lo que a la producción historiográfico-educativa brasileña se refiere. Sobre todo, por sus dimensiones y su diversidad. No obstante, ya en los años 90 advertí la pujanza de dicha producción en contraste con la crisis o declive de la Historia de la Educación que se detectaba en Europa -, comencé a interesarme por ella, y durante mi etapa como miembro del Comité Ejecutivo de la Ische (1996-2000), hice todo lo posible para que en el ámbito latinoamericano, sobre todo en Brasil, fueran creándose sociedades nacionales de Historia de la Educación - no hice más que secundar en tal sentido la labor de Gabriela Ossenbach -, con el fin de que en el a nivel internacional hubiera siempre quien hablara en nombre de la Sociedad de Historia de la Educación de cada país y no, como venía sucediendo, de sí mismo o de un grupo determinado.

Hoy en día, el campo de la Historia de la Educación se halla, en Brasil, no solo plenamente asentado, sino que, además, sigue ofreciendo una vitalidad que, como ya he dicho, contrasta con la de otros países. No pretendo ser pájaro de mal agüero, pero es previsible que la crisis económica y la progresiva mercantilización de la educación supongan un cierto freno en dicha vitalidad. Un proceso similar, en todo caso, al que se está experimentando a nivel internacional. El futuro, también como en otros países, dependerá de la capacidad formativa de quienes actualmente trabajan en este campo y de lo que suceda con la Historia de la Educación como disciplina académica.

Ao finalizar, gostaria de sua opinião sobre os destinos da História da Educação: para onde vai a nossa disciplina? $O$ que poderia ser a agenda para próxima década? Quais perspectivas de pesquisa e que fontes poderiam estar sujeitas a uma maior exploração? Como enfrentar os desafios colocados pela crescente internacionalização do processo de pesquisa?

\footnotetext{
${ }^{8}$ OLIVEIRA, Marcus A. Taborda de; FARIA FILHO, Luciano Mendes de. Antonio Viñao Frago: a crítica da educação como crítica cultural. Revista Educação, São Paulo, 2009, p. 44-59 
La cuestión está planteada de un modo que hace dudar acerca de si la respuesta ha de referirse al futuro que uno desearía o al que uno prevé como posible. Hablar de lo que uno desearía o considera necesario, es un atrevimiento que solo lleva a comprobar, al cabo de varios años, el choque, entre la realidad y los deseos. Además, cualquier intento por parte de una generación, o de los padres, por decirle a la siguiente, a los hijos, por donde deben ir, resulta infructuoso. Cada generación labra su propio campo y abre sus propios caminos. Lo más que se puede decir es que procuren ser intelectualmente algo más honestos e imaginativos de lo que fuimos: o menos deshonestos, rutinarios $y$ cómodos.

En cuanto al futuro previsible, depende de una serie de circunstancias y presiones externas relacionadas con:

a) La carrera académica: puestos de trabajo, modos de formación, evaluación y selección, etc.

b) Las dificultades que plantee la irresoluble tensión que genera un campo disciplinar académicamente ligado al mundo de la pedagogía y ciencias de la educación, pero de naturaleza histórica.

c) Las tecnologías y soportes de la investigación de la cultura electrónica y, ligado a ello, los intereses financieros o de poder académico relativos a la difusión de la investigación, y el papel y criterios seguidos por las agencias nacionales e internacionales encargadas de la evaluación de la actividad investigadora.

d) La existencia, o no, de financiación para investigar unos u otros temas. Un aspecto que explica, por ejemplo, el reciente auge de los estudios patrimoniales y del museísmo pedagógico, en detrimento de otros temas y actividades. La investigación se dirige hacia allí donde hay recursos.

e) La existencia, o no, de recursos y estructuras que favorezcan la movilidad nacional e internacional de los investigadores y la difusión e intercambio de sus trabajos.

Una vez dicho lo anterior, permítaseme, antes de terminar con esta reflexión general, que indique un tema en el que me gustaría que se trabajara en los próximos años, aun sabiendo que es difícil que haya fondos para ello, y que es muy posible que no entre en la agenda de la mayoría de los historiadores de la educación. Pienso que, ante la ligereza con que utilizamos muchos conceptos términos y expresiones, los distintos significados y connotaciones que han tenido, o las apropiaciones e interpretaciones tan diversas de que han sido objeto, que habría que llevar a cabo una historia de aquellos conceptos, términos y expresiones que constituyen el vocabulario básico de nuestra disciplina. Algo al estilo de lo que, en el ámbito de las ciencias sociales, hizo Raymond Williams en Keywords: a vocabulary of culture and society (1976). Es decir, no ofrecer nuestra correcta definición de los mismos, la que cada uno tenga, sino dar cuenta de cómo se han entendido en diferentes momentos, lugares, autores y textos. Una historia de los conceptos y expresiones básicas a lo largo de la Historia de la Educación. Sería un buen modo de que todos, al utilizar uno de dichos términos o expresiones, comenzáramos aclarando al menos el significado o sentido que les atribuimos. 
MARIA HELENA CAMARA BASTOS é professora na Pontifícia Universidade Católica do Rio Grande do Sul. Professora visitante na Università degli studi di Macerata/Itália.

Endereço: Rua Eng. Álvaro Nunes Pereira, 340/906 - 90570-110 - Porto Alegre - RS - Brasil.

E-mail: $\underline{\text { mhbastos1950@gmail.com. }}$

Recebido em 12 de setembro de 2016.

Aceito em 27 de outubro de 2016. 